\title{
Oral feeding for infants and children receiving nasal continuous positive airway pressure and high flow nasal cannula: a systematic review
}

\author{
Angie Canning $^{1 *}$ (D), Sally Clarke ${ }^{2}$, Sarah Thorning ${ }^{3}$, Manbir Chauhan ${ }^{4}$ and Kelly A Weir ${ }^{5,6}$ (D)
}

\begin{abstract}
Background: The aim of this systematic review was to determine whether introduction of oral feeding for infants and children receiving nasal continuous positive airway pressure (nCPAP) or high flow nasal cannula (HFNC) respiratory support facilitates achievement of full oral feeding without adverse effects, compared to no oral feeding (NPO; nil per oral) on CPAP or HFNC.

Methods: A protocol was lodged with the PROSPERO International Prospective Register of Systematic Reviews. We searched Medline, Embase, CINAHL, CENTRAL and AustHealth from database inception to 10th June 2020. Study population included children (preterm to $<18$ years) on nCPAP or HFNC who were orally feeding. Primary outcomes included full or partial oral feeding and oropharyngeal aspiration. Secondary outcomes examined adverse events including clinical signs of aspiration, aspiration pneumonia and deterioration in respiratory status.

Results: The search retrieved 1684 studies following duplicate removal. Title and abstract screening identified 70 studies for full text screening and of these, 16 were included in the review for data extraction. Methods of noninvasive ventilation (NIV) included nCPAP $(n=6), \operatorname{nCPAP}$ and HFNC $(n=5)$ and HFNC $(n=5)$. A metanalysis was not possible as respiratory modes and cohorts were not comparable. Eleven studies reported on adverse events. Oral feeding safety was predominantly based on retrospective data from chart entries and clinical signs, with only one study using an instrumental swallow evaluation (VFSS) to determine aspiration status.

Conclusions: Findings are insufficient to conclude whether commencing oral feeding whilst on nCPAP or HFNC facilitates transition to full oral feeding without adverse effects, including oropharyngeal aspiration. Further research is required to determine the safety and efficacy of oral feeding on CPAP and HFNC for infants and children.
\end{abstract}

Trial registration: PROSPERO registration number: CRD42016039325.

Keywords: Oral feeding, nCPAP, HFNC, Pediatric, Swallowing

\footnotetext{
* Correspondence: angie.canning@health.qld.gov.au

'Speech Pathology, Gold Coast University Hospital, Gold Coast Health, Gold Coast, Australia

Full list of author information is available at the end of the article
}

(c) The Author(s). 2021 Open Access This article is licensed under a Creative Commons Attribution 4.0 International License, which permits use, sharing, adaptation, distribution and reproduction in any medium or format, as long as you give appropriate credit to the original author(s) and the source, provide a link to the Creative Commons licence, and indicate if changes were made. The images or other third party material in this article are included in the article's Creative Commons licence, unless indicated otherwise in a credit line to the material. If material is not included in the article's Creative Commons licence and your intended use is not permitted by statutory regulation or exceeds the permitted use, you will need to obtain permission directly from the copyright holder. To view a copy of this licence, visit http://creativecommons.org/licenses/by/4.0/ The Creative Commons Public Domain Dedication waiver (http://creativecommons.org/publicdomain/zero/1.0/) applies to the data made available in this article, unless otherwise stated in a credit line to the data. 


\section{Background}

The use of non-invasive ventilation (NIV), including nasal continuous positive airway pressure (nCPAP) and high flow nasal cannula (HFNC), has increased over the last two decades as primary or step-down respiratory therapies for infants and children with acute and chronic respiratory conditions [1-3]. They provide support for infants in neonatal intensive care units (NICU) with respiratory distress syndrome and bronchopulmonary dysplasia (BPD)/chronic neonatal lung disease (CNLD) $[1,4]$, and children in pediatric intensive care units (PICU) for treatment of acute illnesses such as bronchiolitis and pneumonia; and can reduce the need for invasive ventilation $[5,6]$. However, the impacts of nCPAP and HFNC on oral feeding and swallowing are unknown [5, 7].

Historically, infants and children receiving nCPAP and HFNC were kept nil per oral and received tube feedings only, due to concerns regarding impacts on swallow safety (considered to be at a higher risk of oropharyngeal aspiration: fluid/food entering the airways below the level of the vocal folds) and cardiorespiratory stability [8-11]. HFNC increases pharyngeal pressures, which may affect laryngeal closure, pharyngeal sensory responses, and in turn, airway protection mechanisms [7, 11-14]. nCPAP is known to impact the timing and frequency of the swallow reflex in adults [15]. Thus, potential aspiration during oral feeding whilst receiving nCPAP and HFNC could increase the duration of respiratory support requirement, increase hospital length of stay and negatively impact feeding and respiratory outcomes.

As non-invasive respiratory therapies provide access to the mouth, oral feeding for infants and children receiving nCPAP and HFNC is increasingly being provided. In the preterm population the requirement for nCPAP or HFNC may coincide with infants' developmental readiness for oral feeding. Therefore oral feeding experiences are provided in an attempt to support neurodevelopmental outcomes, facilitate transition to full oral feeding and reduce length of stay $[8,10,16,17]$. For infants and children with acute respiratory illness, poor nutrition is thought to increase length of PICU stay, therefore oral feeding may be provided to optimise nutrition, reduce length of stay and for comfort $[9,13]$.

A recent survey of practice of NICUs and PICUs in Australian and New Zealand reported that most units surveyed do feed on NIV, more frequently on HFNC, and with use of strategies including monitoring stability and reducing pressure/flow rate during oral feeding. The primary reason for not orally feeding on NIV was that the aspiration risk is unclear The survey reported high variability in feeding practices, differing clinical opinion and a lack of evidence-based clinical guidelines regarding oral feeding for this cohort [18]. Therefore this systematic review aimed to determine if oral feeding for infants and children receiving nCPAP and HFNC facilitates full oral feeding without adverse effects (including oropharyngeal aspiration).

\section{Methods}

The study protocol was conducted in accordance with the Preferred Reporting Items for Systematic Reviews and Meta-Analyses (PRISMA) Statement and lodged with the PROPERO International Prospective Register of Systematic Reviews (CRD42016039325) [19].

A comprehensive search was conducted by a medical librarian (ST) using the following databases: Medline (Ovid), Embase (Elsevier), CINAHL (Ebsco), The Cochrane Central Register of Controlled Trials (CENT RAL) and AustHealth (Informit) from database inception to 10th June 2020. Manual searching of reference lists of studies retrieved for data extraction was undertaken. There was no restriction on publication date or language. The search strategy included the following keywords or Medical Subject Headings (MeSH) terms: 1) suck or feed or oral or bottle or breast or nipple or infant feeding; and; 2) high flow and nasal cannula or nasal prong or oxygen; cpap or ncpap or bcpap or peep or positive end expiratory pressure or continuous positive airway pressure or positive end expiratory pressure. The full search strategy is documented in the PROSPERO protocol [19].

Included studies met the following criteria: 1) pediatric population (birth to $<18$ years); 2) participants received oral feeding/nutritive swallowing (i.e., breast, bottle feeding, cup drinking, solids intake); 3 ) participants received nCPAP or HFNC therapy at the time of oral feeding/nutritive swallowing; 4) study types included randomised control trials, control trials, cohort studies, case series and case reports. Grey literature was not included.

The criteria were kept deliberately broad to encompass children at different ages and stages of their feeding development, as this reflects the children who we see clinically at our tertiary institution. Oral feeding was defined as any amount of fluid/food taken by mouth. Studies were excluded if they were adult populations ( $\geq 18$ years of age); received only low flow nasal cannula (LFNC) support or invasive ventilation; or participants were nil per oral (parenteral/tube feeding only).

Three primary outcomes were established:

1) Full oral feeding (receiving all nutrition and hydration by mouth and no longer receiving tube/ parenteral feeding)

2) Partial oral feeding (defined as 'oral feeding with supplemental tube/parental feeding' or 'oral feeding without full oral feeding' reported as an outcome) 
3) Oropharyngeal aspiration, as observed on instrumental assessment (videofluoroscopic swallow study or fiberoptic-endoscopic evaluation of the swallow)

Secondary outcomes examined adverse effects including:

1) Clinical signs of oropharyngeal aspiration

2) Aspiration pneumonia or use of antibiotics for clinically suspected aspiration pneumonia

3) Deterioration in respiratory status or respiratory distress (increased work of breathing/oxygen requirements, oxygen desaturations, chest $\mathrm{x}$-ray findings)

4) Oral aversion/feeding refusal

5) Behavioural responses (e.g. gagging/disengagement/ refusal cues)

6) Gastro-oesophageal reflux

7) Death

Initial screening of the title, abstract and keywords, and full text reviews were performed by two authors (AC,SC) according to the inclusion and exclusion criteria using Covidence [20]. Disagreements were resolved by consulting a third author (KW) and consensus reached. Two review authors (AC,KW) independently performed data extraction and discrepancies resolved through discussion or with a third author (SC).

Quality assessment of the included studies was undertaken independently by two reviewers $(\mathrm{AC}, \mathrm{KW})$ and disagreements resolved through discussion. Risk of bias was determined using the Cochrane Risk of Bias in Nonrandomized Studies (ROBINS-1) [21] and The Cochrane Risk of Bias (ROB-2) [22] tools. Publication bias was not assessed due to the small number of trials.

\section{Results}

Database searching retrieved 1684 records after duplicates were removed. One further record was added through reference list searching. Title and abstract screening identified 70 studies for full text screening. Full text screening identified 16 studies that met eligibility criteria for inclusion in the review and 54 studies were excluded. See Fig. 1 for PRISMA flow diagram.

\section{Study characteristics}

See Table 1 for a summary of study characteristics.

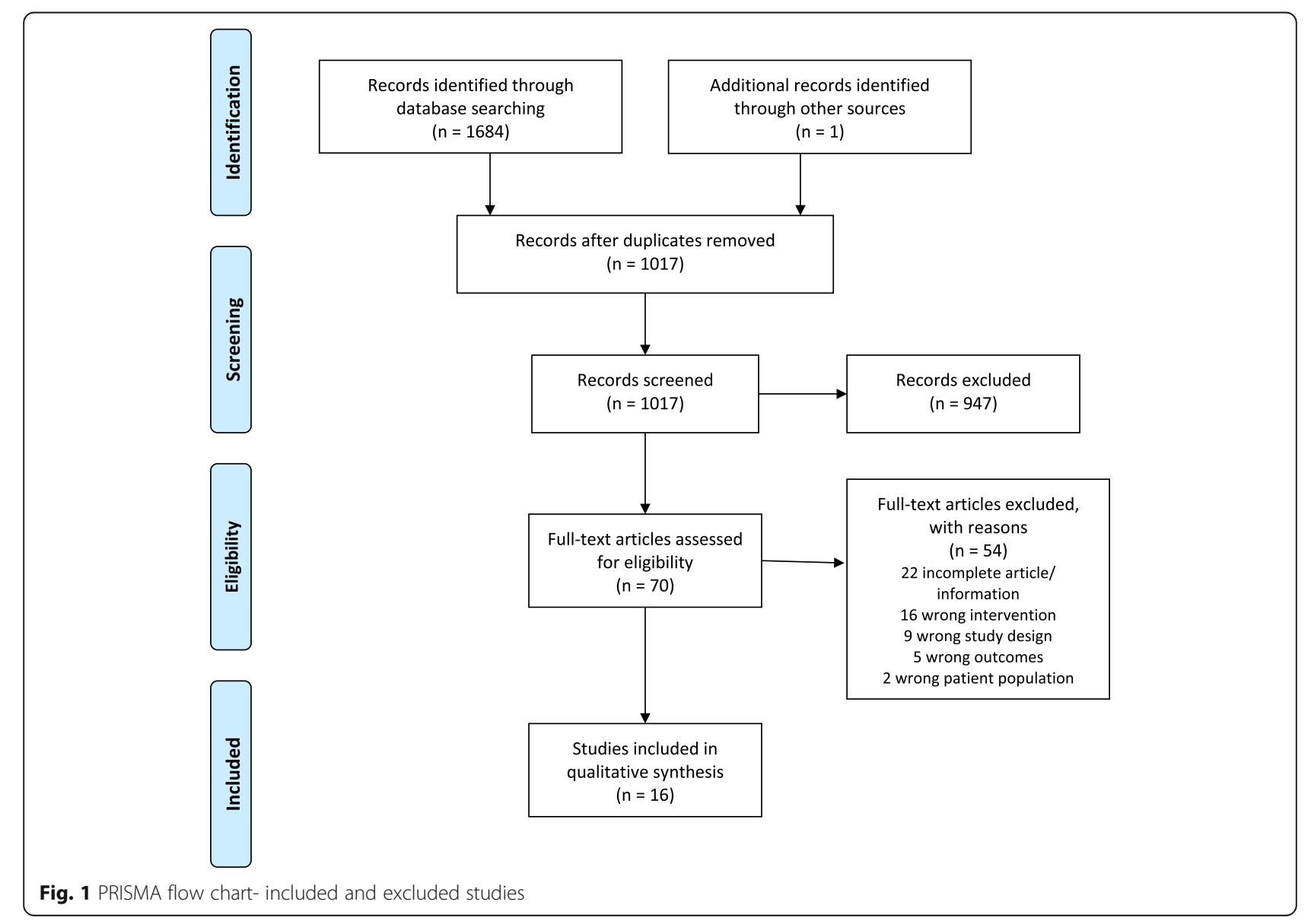




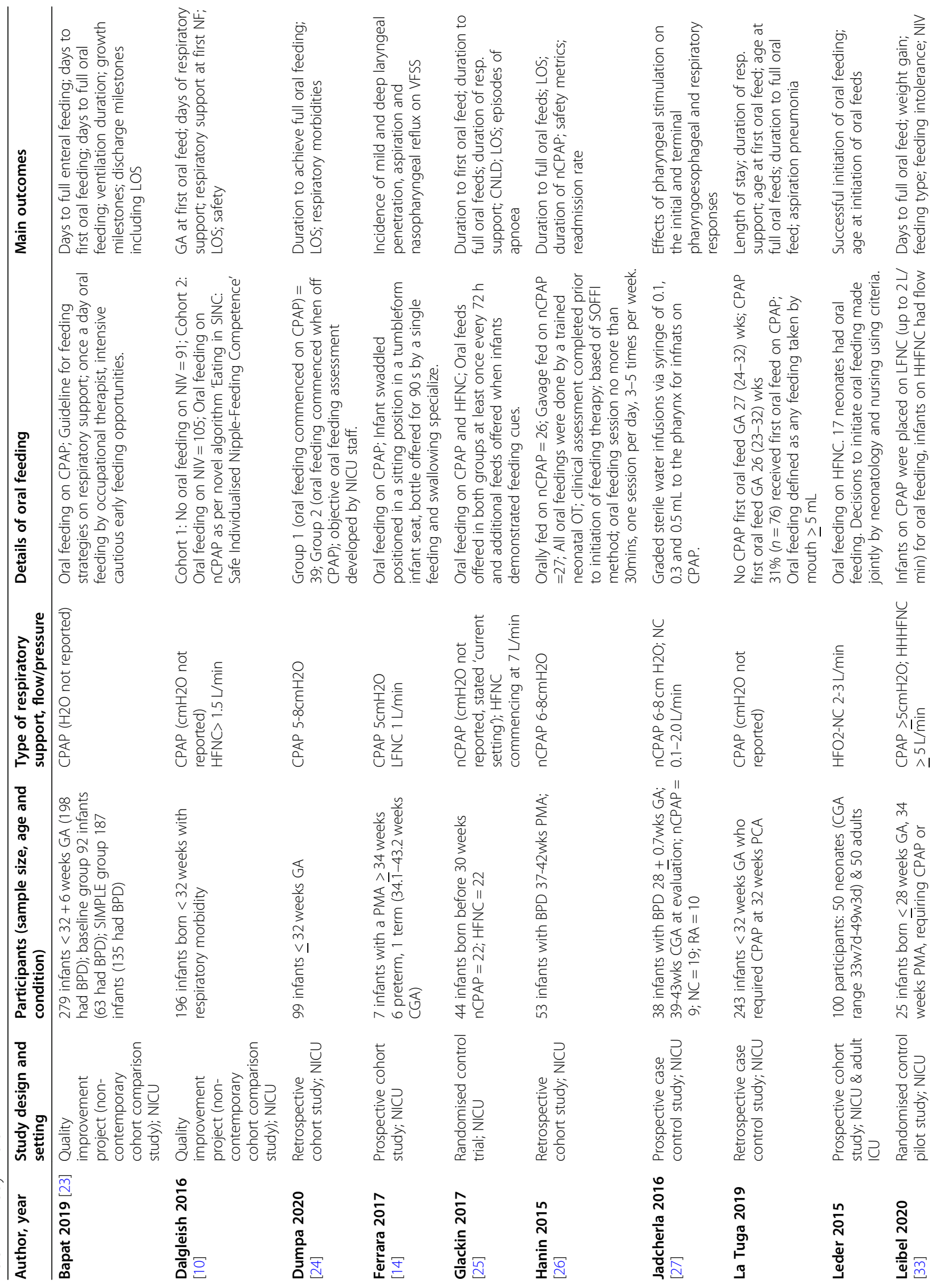




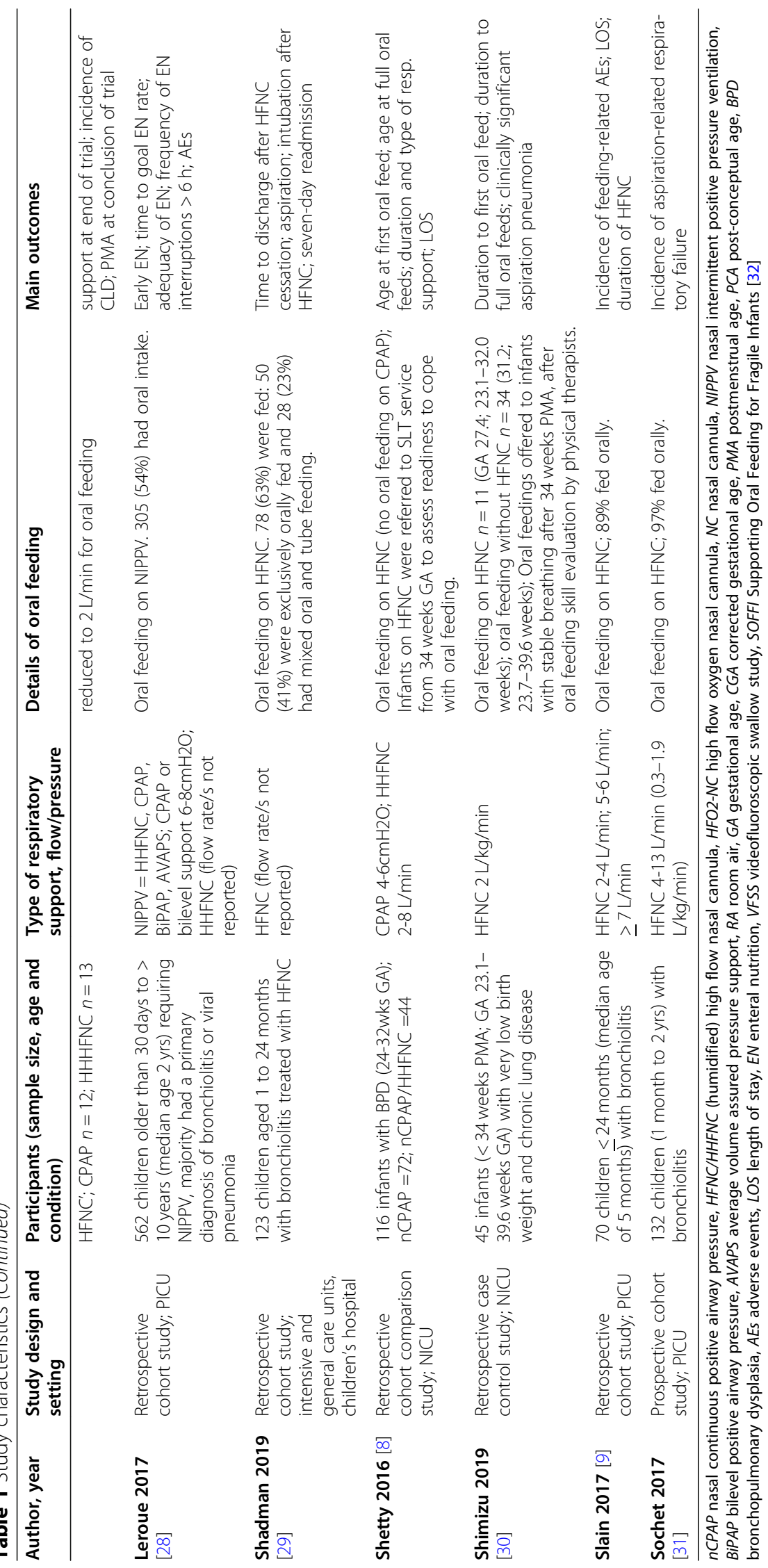


Five retrospective cohort studies [9, 24, 26, 28, 29], three prospective cohort studies [14, 16, 31], two randomised control studies [25, 33], two retrospective case control studies [30,34], two quality improvement projects (non-contemporary cohort comparison studies) $[10,23]$, one prospective case control study [27] and one retrospective cohort comparison study [8] were included in this review.

Study sample sizes ranged from seven to 562 participants. In one study [16] involving both adult and neonatal patients, only the neonatal data was included in the review. Twelve studies included participants from NICUs with five studies $[8,23,26,27,30]$ including infants with a diagnosis of CNLD/BPD, whilst seven studies $[10,14,16,24,25,33,34]$ included patients with respiratory morbidity requiring NIV with no further diagnostic specification. Three studies [9, $28,31]$ were from PICU settings and one study was from both intensive and general care units in a children's hospital [29]. Three of these studies included children aged 0 to 24 months with bronchiolitis [9, $29,31]$ and one study $(n=562)$ included children aged 30 days to 10 years with a range of diagnoses including bronchiolitis (48\%), viral pneumonia (16\%) and status asthmaticus (18\%) [28].

Six studies $[14,23,24,26,34]$ included participants receiving nCPAP only, with three studies reporting on nCPAP pressures. In five studies $[9,16,29-31]$ participants received HFNC only, with flow rates reported in four studies. Five studies $[8,10,25,28,33]$ included participants receiving $\mathrm{nCPAP}$ and HFNC.

\section{Main outcomes}

Main outcomes included age at/duration to first oral feed, age at/duration to full oral feeds, adequacy and frequency of enteral nutrition, adverse events, duration of respiratory support, length of stay, number of participants discharged with tube feeding, type of feeding at discharge and readmission rate.

\section{Quality of individual studies}

For the 14 included non-RCTs, three studies were judged to have a moderate risk, 10 studies a serious risk and one study a critical risk of bias. Due to the nature of the research designs, no studies were judged to have a low risk of bias. Investigators were not blinded and allocation to intervention was sometimes based on physician judgment. One included RCT had an overall judgment of 'some concerns' due to risk of bias arising from deviations from the intended interventions, and the other included RCT was judged to have a low risk of bias. Refer to Figs. 2 and 3 for risk of bias summaries.

\section{Results of individual studies}

The following information was extracted from the included studies against the outcome measures established for this review.

\section{Analysis of primary outcomes}

See Table 2 for a summary of primary outcomes.

\section{1) Full oral feeding}

Of the 16 included studies, 12 reported on full or exclusive oral feeding $[8,9,23-26,28-31,33,34]$. Three studies $[24,26,34]$ compared the duration to full oral feeds for infants initiating oral feeding whilst on CPAP versus those who commenced oral feeding after ceasing CPAP. Hanin [26] found that infants who initiated oral feeding while on CPAP achieved oral feeding milestones at an earlier postmenstrual age (PMA), however Dumpa [24] reported no significant difference (longer duration if started oral feeding on CPAP but achieved full oral feeding at same PMA) and LaTuga [34] reported that infants who started oral feeding on CPAP took longer to attain full oral feeding.

One study reported on oral feeding outcomes for infants on CPAP only. Bapat [23] reported on duration to full oral feeding for preterm infants with BPD who participated in a quality improvement project to enhance feeding milestones and found that infants with mild to moderate (but not for severe) BPD achieved full oral feeds earlier on their SIMPLE feeding program (median 81 days of life) vs baseline group (median 84 days of life) $(p<0.05)$.

Four studies examined oral feeding on HFNC only [9, 29-31], with one study [30] comparing duration to full oral feeds for preterm infants initiating oral feeding whilst on HFNC versus infants initiating oral feeding while not on HFNC. Shimizu [30] found that there were similar ages for achieving full oral feeding between the two groups.

Three studies $[8,25,33]$ compared the duration to full oral feeding for infants supported by nCPAP versus HFNC. Glackin [25] studied infants who were orally fed on both CPAP and HFNC and reported the number of days to achieve full oral feeding was not significantly different between the two cohorts. In Shetty [8] infants were fed on HFNC only and the age to achieve full oral feeding was not found to be significantly different in either group, however a sub-analysis of infants receiving NIV beyond 34 weeks PMA showed that full oral feeding was achieved significantly earlier in the nCPAP-thenHFNC group. In Leibel [33] infants on CPAP were placed on LFNC $(<2 \mathrm{~L} / \mathrm{min})$ to orally feed and infants on HFNC had their flow reduced to $2 \mathrm{~L} / \mathrm{min}$ to orally feed. Infants randomised to the HFNC group reached full oral feeds earlier. 


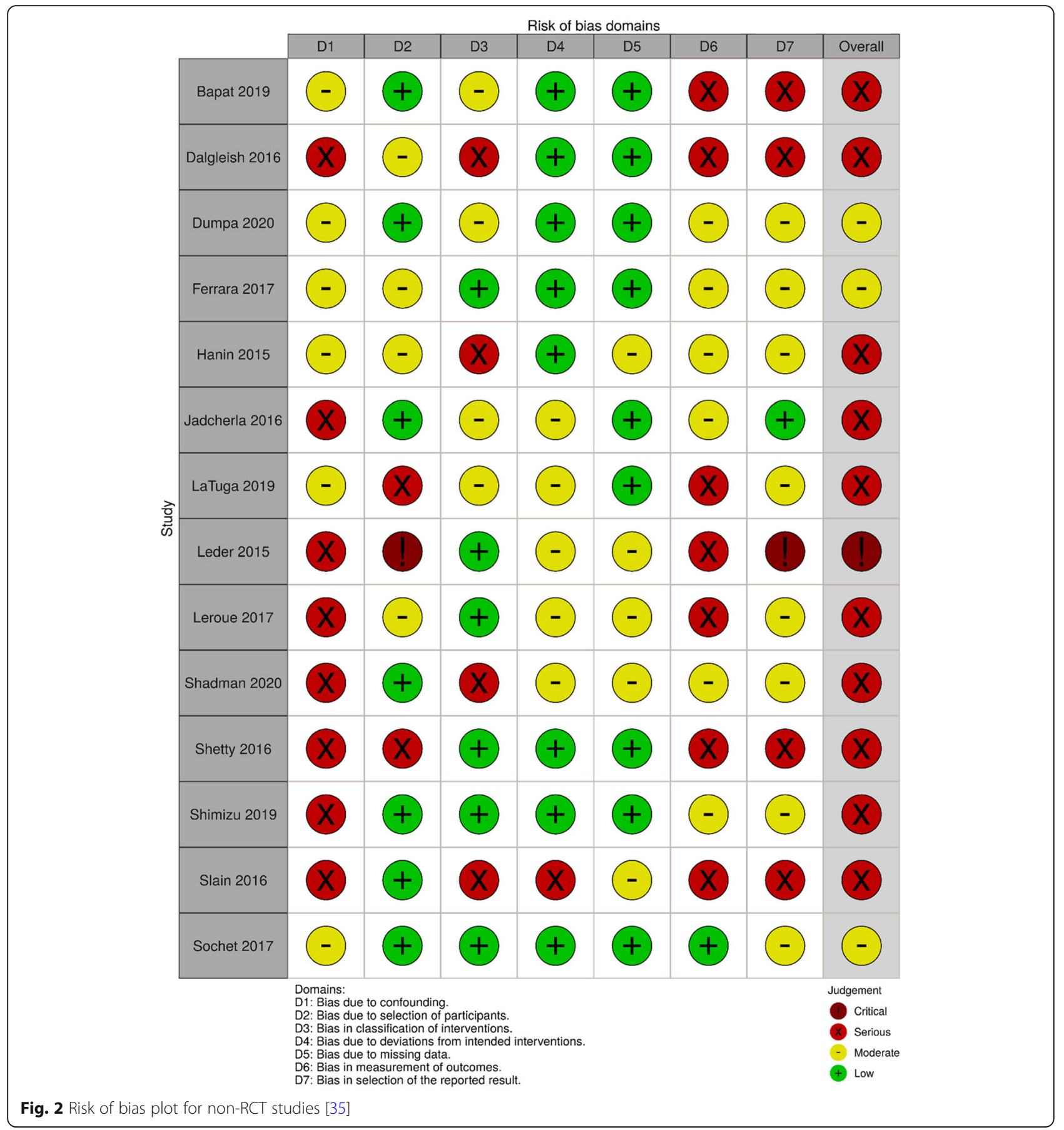

Four studies from PICU/pediatric hospital settings $[9,28,29,31]$ reported only on the number of children receiving oral nutrition on NIV rather than duration to full oral feeds.

2) Partial oral feeding

Eight studies $[8,10,16,23-25,30,34]$ reported age at first oral feed. An additional four studies reported on some degree of oral feeding while receiving NIV, such as $50 \%$ of total fluid intake, mixed oral and tube feedings or small volumes via syringe.

3) Oropharyngeal aspiration, as observed on instrumental assessment

One study [14] used an instrumental swallow evaluation to assess seven infants (mean PMA 38.1 weeks) 


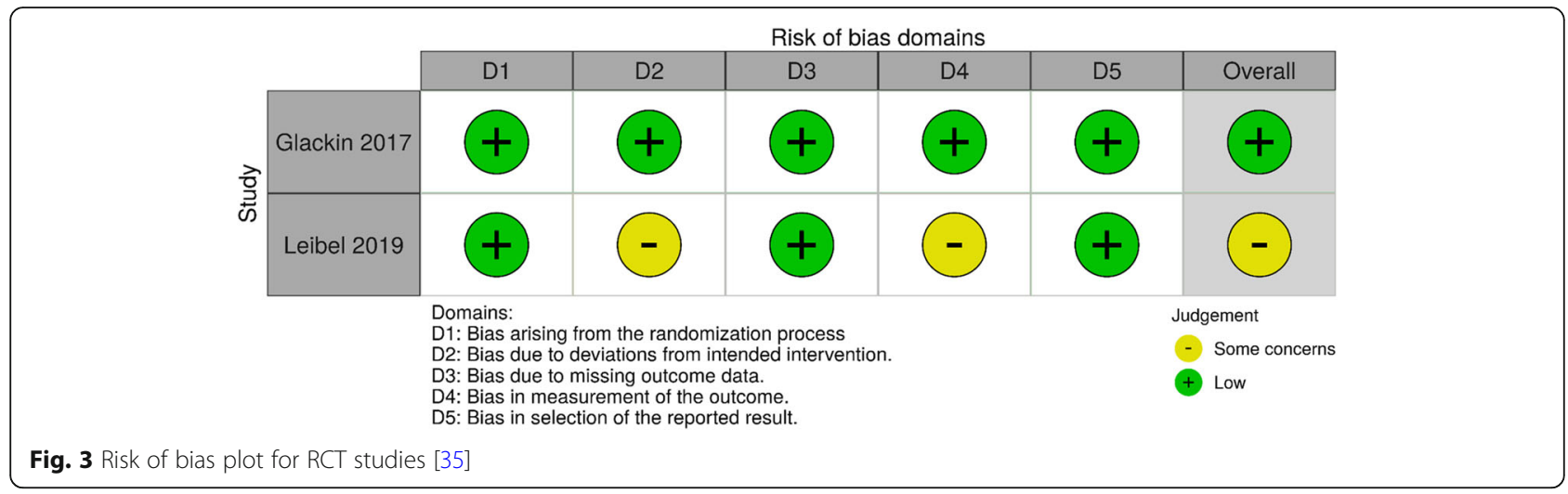

orally feeding on nCPAP. Ferrara [14] utilised VFSS to assess for aspiration during 20 swallows under two conditions: on-nCPAP $(5 \mathrm{cmH} 20)$ and off-nCPAP $(1 \mathrm{~L} / \mathrm{min}$ LFNC). Infants demonstrated significantly more frequent episodes of deep laryngeal penetration (43.7\% vs $25.3 \%)$ and aspiration (33.5\% vs $14.6 \%$ ) when on-nCPAP versus off-nCPAP respectively. The remaining 15 studies did not utilise instrumental evaluation of the swallow to confirm oropharyngeal aspiration status.

\section{Analysis of secondary outcomes}

Secondary outcomes examined adverse events (AEs) and these were reported by 11 studies.

See Table 3 for a summary of secondary outcomes 1-3.

1) Clinical signs of oropharyngeal aspiration

Clinical signs suggestive of oropharyngeal aspiration (OPA) include coughing, choking, noisy or wet breathing, wet vocalisations, wheeze, recurrent pneumonia, gagging, congestion, tachypnoea, bradycardia, apnoea, colour changes, oxygen desaturations and voice changes during and/or after feeds [36, 37]. Six studies [9, 10, 16, 25, 26, 33 reported on clinical signs of aspiration, including the incidence of specific clinical signs or general statements.

2) Aspiration pneumonia or use of antibiotics for clinically suspected aspiration pneumonia

Nine studies $[10,25,26,28-31,33,34]$ reported on the incidence of aspiration pneumonia and/or use of antibiotics for clinically suspected aspiration pneumonia.

3) Decrease in respiratory status/respiratory distress

Eight studies [9, 10, 25, 26, 28, 30, 31, 33] reported on the incidence of deterioration in respiratory status or respiratory distress in response to oral feeding on nCPAP or HFNC.
4) Other secondary outcomes

Two studies reported on behavioural responses during oral feeding on nCPAP or HFNC. Hanin [26] reported on the frequency of oral feedings on nCPAP that were ceased in response to 'more than one episode of coughing or gagging' $(0.4 \%, n=1$, total oral feedings $=218)$, however the descriptors were nonspecific/unclear if coughing or gagging. Dalgleish [10] reported that no infant exhibited symptoms of 'ongoing or recurring distress related to nipple feeding opportunities'.

Two studies [23, 29] reported on readmissions and one study [31] reported death as an outcome, stating zero mortality for children with acute viral bronchiolitis on HFNC receiving enteral nutrition. No studies reported on oral aversion/feeding refusal or gastroesophageal reflux.

\section{Additional analysis}

A meta-analysis was not possible due to significant heterogeneity between included studies.

\section{Discussion}

This study aimed to systematically review the literature to evaluate whether oral feeding on nCPAP and HFNC facilitates transition to full oral feeds without adverse effects. The findings are insufficient to conclude whether commencing oral feeding whilst on nCPAP or HFNC facilitates transition to full oral feeding and the risk of adverse events including oropharyngeal aspiration is unclear.

Duration to full oral feeding for participants on nCPAP or HFNC was associated with gestational age (GA) at birth and severity of respiratory disease, which also reflects literature for children without NIV support. The development of oral feeding skills and duration to full oral feeding are known to be related to GA at birth for preterm children, with extremely and very preterm infants achieving full suck feeds later [38, 39]. Infants and children with respiratory disease are also at 


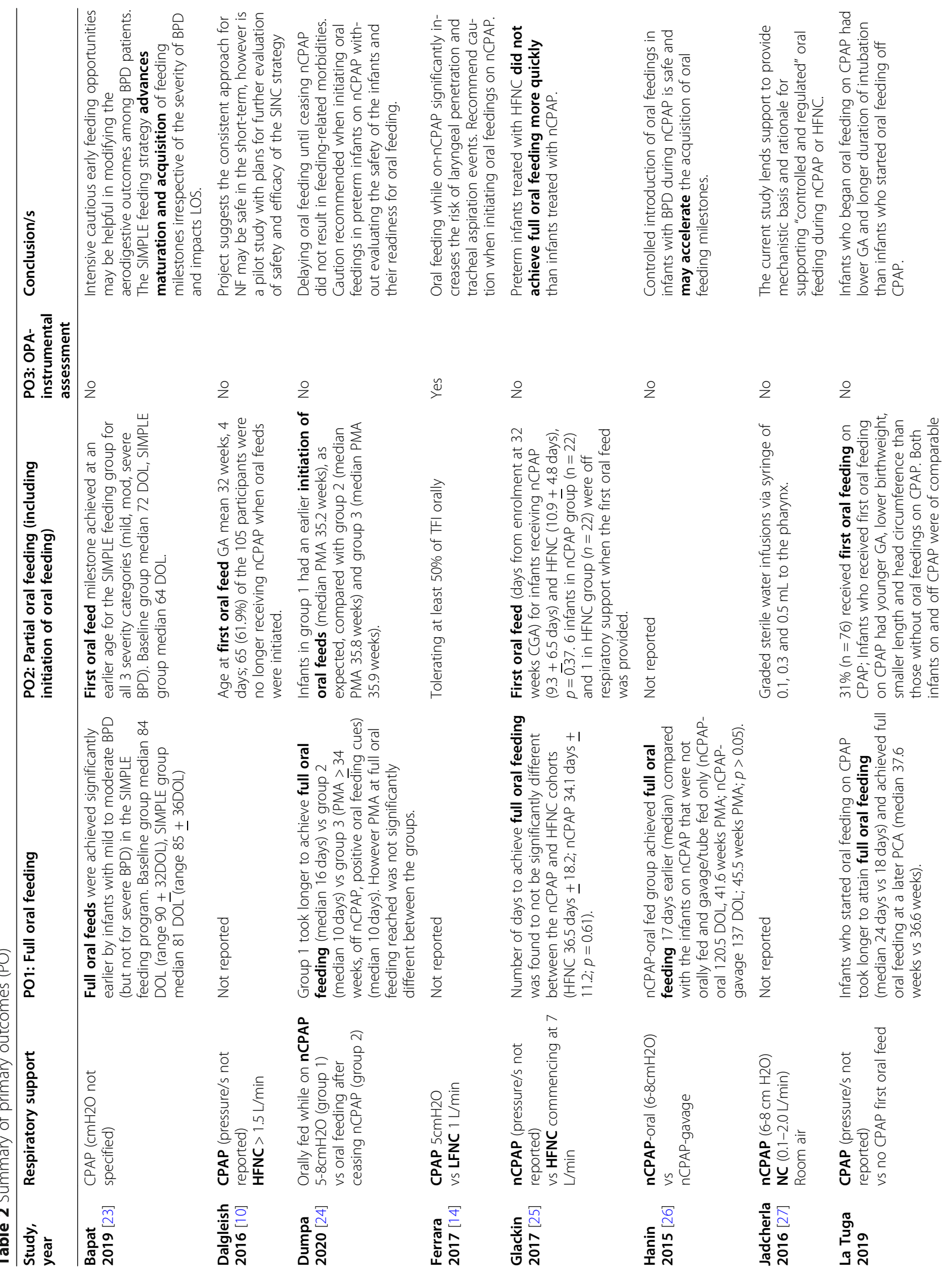




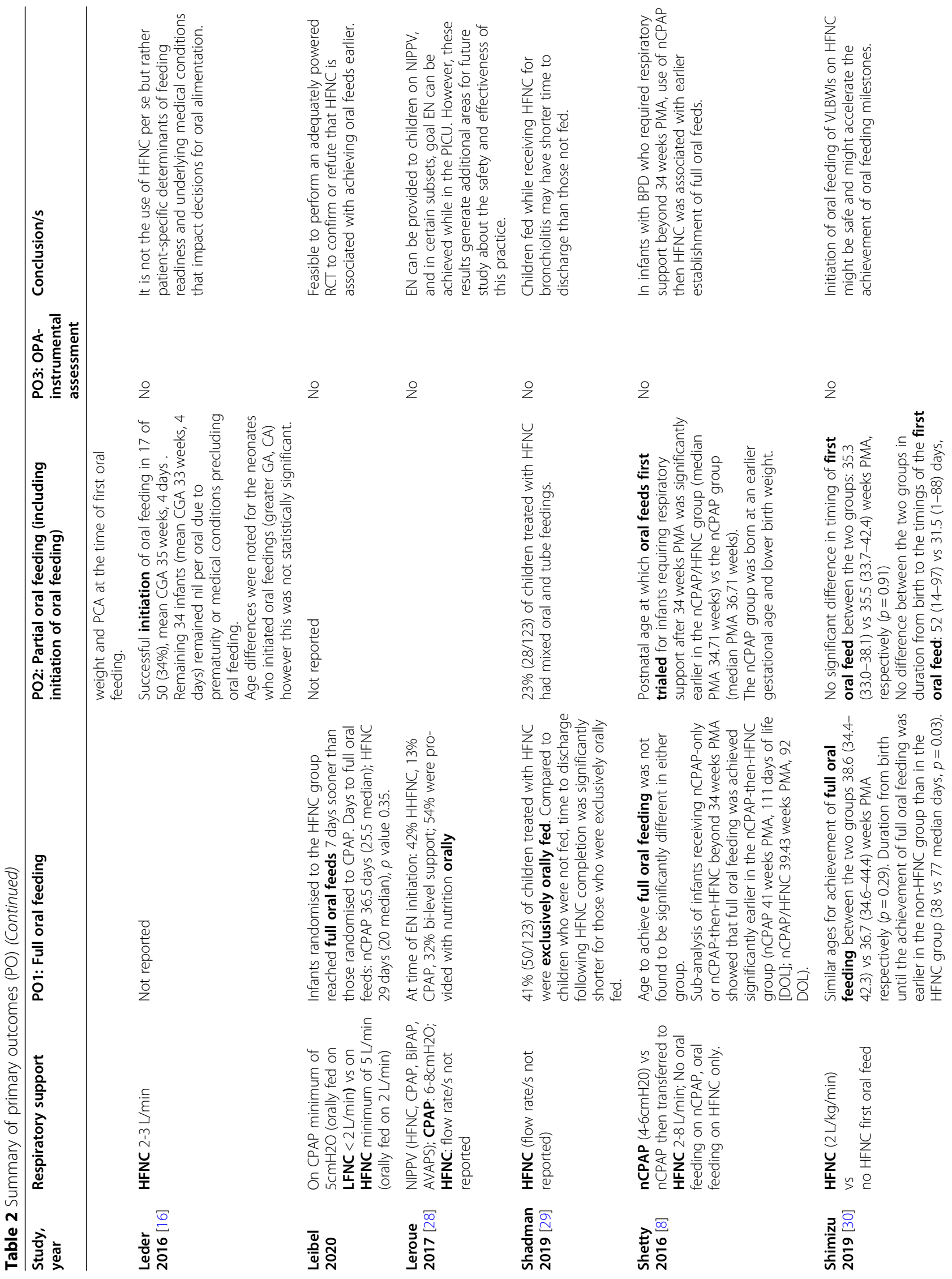




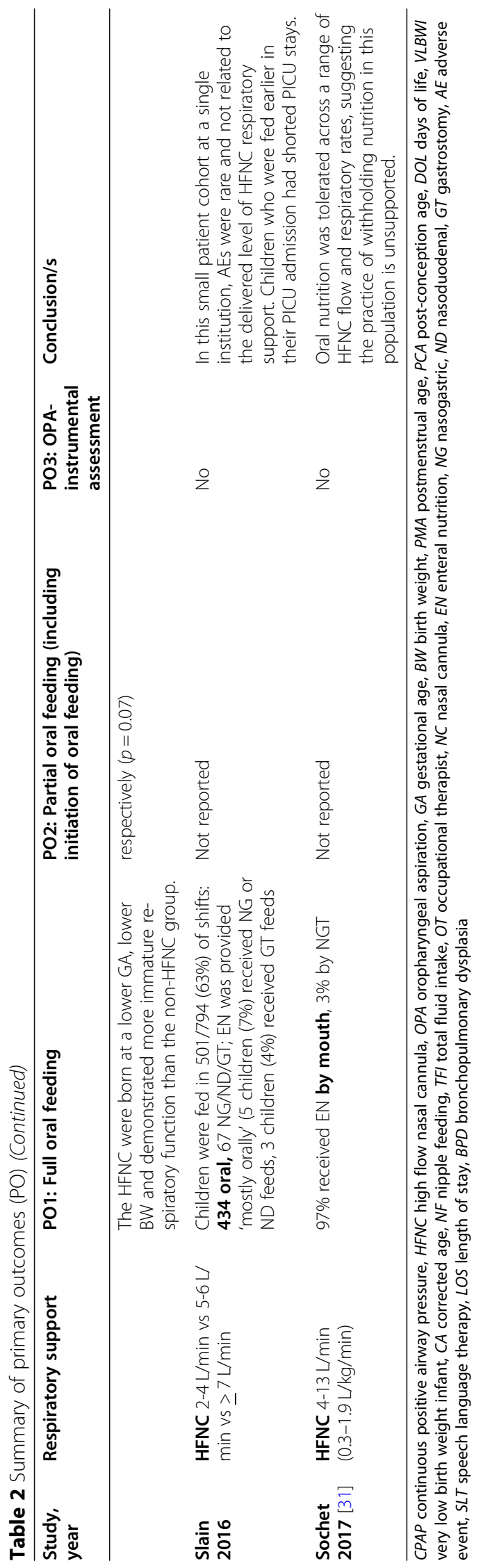




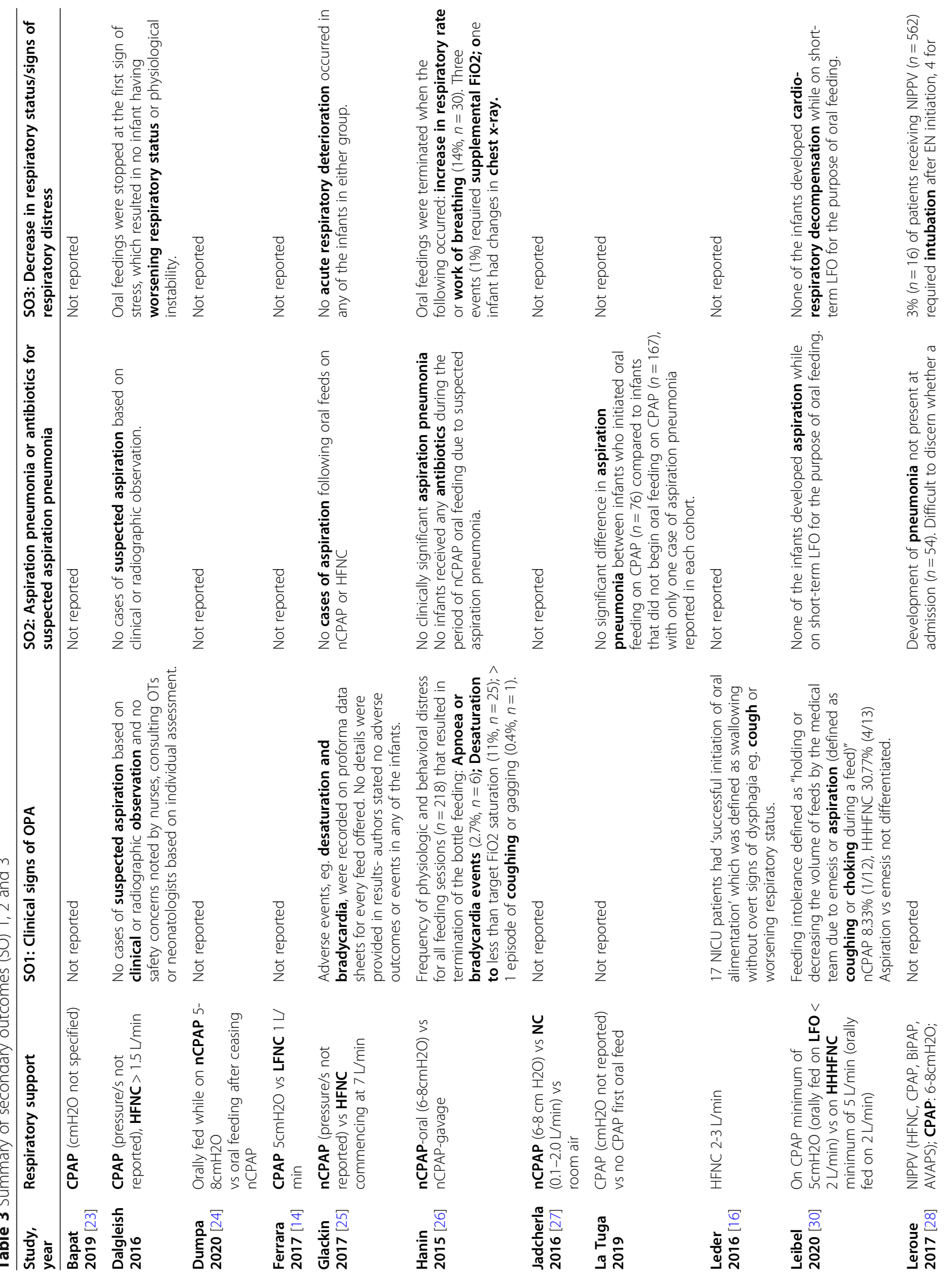




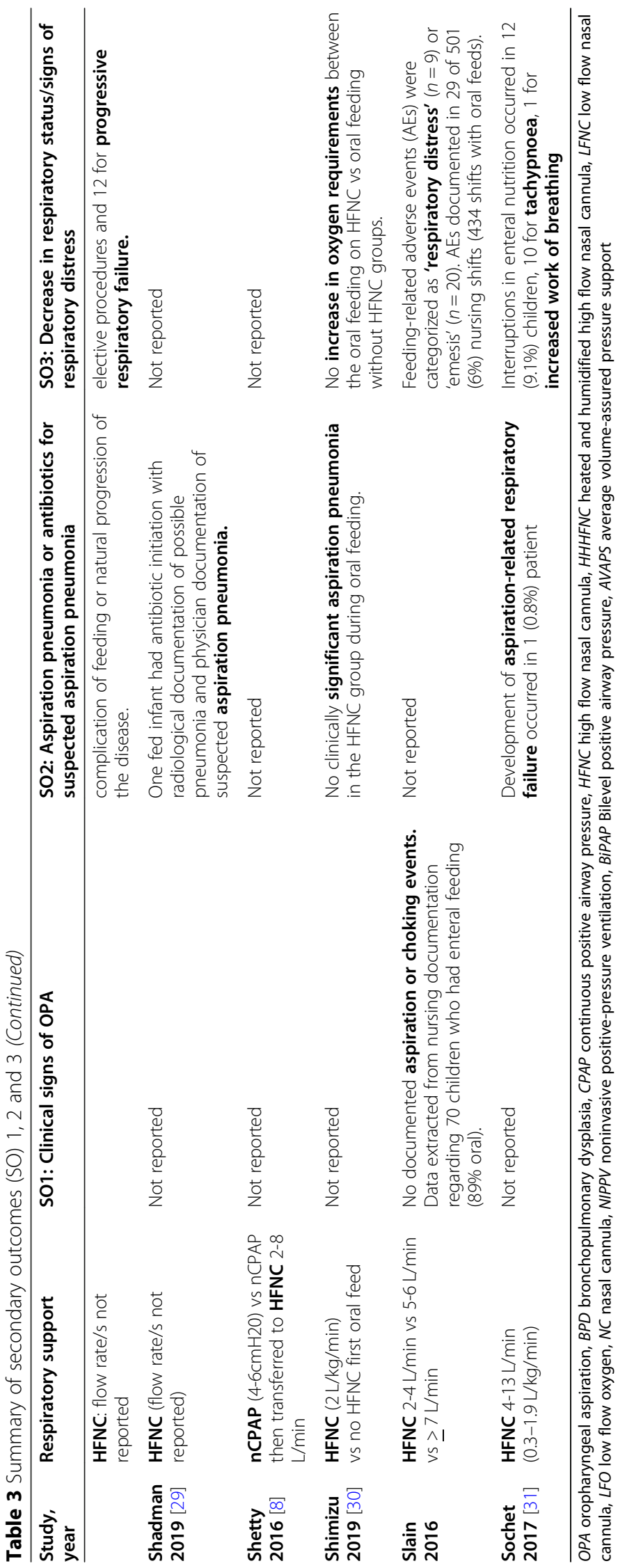


increased risk of feeding difficulties, without the added complication of NIV [40]. Reduced suck-swallowbreathe coordination, poor feeding efficiency and endurance, weak sucking pressures and difficulties ingesting adequate intake have been reported in this population [41, 42]. Studies matching infants requiring NIV with those no longer requiring NIV are likely comparing cohorts with different respiratory disease severity and therefore feeding outcomes will likely be different, regardless of the use of NIV.

Reported adverse events (AEs) due to oral feeding on NIV varied. AEs during oral feeding may include physiological, respiratory and behavioural responses including desaturation, bradycardia, increase in work of breathing or coughing/choking. Information regarding AEs were mostly obtained retrospectively via chart review or a lack of documented problems was reported, eg. 'no documented aspirations or choking events' [9] presenting opportunities for missed data. The majority of studies did not specify tools used to record AEs or staff training in recognising AEs, therefore reported rates of AEs may be low.

Only a small number of studies reported on the use of supportive feeding practices, including assessing feeding readiness, reading infant cues, stopping a feed at the first sign of stress or physiologic instability, use of modified teat flow rate, feeding the infant in a sidelying position and use of external pacing. Oral feeding strategies are beneficial to support physiological stability and reduce the risk of cardiorespiratory events and infant stress/disengagement during oral feedings in preterm or unwell infants [32]. Lack of implementation of supportive strategies may contribute to AEs and increase risk of aspiration during oral feeding on NIV.

This review identified a lack of studies utilising instrumental assessment tools for assessing swallow safety. While VFSS and FEES are considered gold standards for evaluating aspiration, only one study [14] used VFSS to determine aspiration status of infants orally feeding while on nCPAP. Based on their preliminary findings of increased laryngeal penetration and aspiration in children on nCPAP, Ferrera and colleagues reported that their ethics committee discontinued the trial, and their institutional practice was changed to have children placed NPO whilst receiving nCPAP support. No studies to date have utilitised VFSS or FEES to assess aspiration status of infants and children orally feeding while on HFNC. Most studies reported on clinical signs of aspiration only. Signs and symptoms of aspiration are known to be age dependent, with children experiencing high rates of silent aspiration, and clinical evaluation having lower sensitivity in detecting aspiration $[36,37]$ thus likely to underreport the true incidence of aspiration. In addition, premature infants and previously healthy infants with RSV bronchiolitis are known to be at increased risk of aspiration $[40,43]$ in the absence of NIV, so it can be difficult to determine if the clinical signs of aspiration are related to the underlying condition or to the presence of NIV. Some studies reported on the use of antibiotics for clinically suspected aspiration pneumonia or use of chest $\mathrm{x}$-ray to assess for aspiration, which has poor sensitivity as a diagnostic tool for microaspiration [44]. The clinical response to aspiration can depend on the frequency of aspiration, volume and type of material aspirated and health status of the patient [45]. Undetected aspiration may prolong respiratory support requirements and lead to negative outcomes such as oral aversion and respiratory morbidities.

In addition, studies varied in their definition of HFNC. A 2014 Cochrane review stated that 'high flow' has not been well described in the literature, and defined HFNC in children as having flow rates of $\geq 2 \mathrm{~L} / \mathrm{min}$ [46]. During HFNC therapy, mean nasopharyngeal pressure increases as flow increases but decreases with infant weight [ 7 , 12]. Flow rates can therefore have different impacts on children depending on their size, so HFNC is best described as a weight-adjusted flow rate (ie. L/min/ $/ \mathrm{kg}$ ). Use of this unit would allow more accurate comparison between cohorts and to determine if there is a correlation between flow rate and adverse events.

Finally, another factor of consideration with HFNC is the effect of mouth position on oral and pharyngeal pressures. Wilkinson et al. [7] demonstrated that mouth position during HFNC had little effect on pharyngeal pressures, likely due to nasal leak, however Kubicka et al. [47] reported that the amount of pressure generated during HFNC was related to the degree of mouth opening. A sealed oral cavity during suck feeding therefore may have the potential to further increase pharyngeal pressures and impact swallow safety, however this may be more variable for older children and the type of utensils being used for eating and drinking.

Limitations of this review include a small number of studies retrieved from our search, small sample sizes in some included studies, the retrospective nature of many studies and only one study utilising instrumental assessment of the swallow to determine aspiration status (on nCPAP). Given the lack of clear determination of aspiration status when orally feeding whilst receiving nCPAP or HFNC, it is difficult to provide clear guidance as to what should be best practice clinical care for infants and children. There is a clear need for future prospective research of high quality cohort or controlled trials to determine aspiration status using instrumental evaluation. Additionally, future research should evaluate the range of clinical practices that are provided to infants and children of different ages and with different underlying respiratory conditions. Future studies should include a 
comparison of both and utilizing instrumental evaluation such as FEES or VFSS to determine aspiration, as well as duration on respiratory support and length of stay. Older infants and children (often with an underlying respiratory infectious disease) receiving nCPAP or HFNC may be changed to low flow supplemental oxygen for oral feeding (due to concerns about aspiration risk) and returned to their high flow/pressure respiratory support after the feed/mealtime. We have no way of knowing whether this practice supports or negatively impacts on aspiration risk for these children. Others may be fed, or eat and drink, whilst on their respiratory support for mealtimes. These scenarios are commonly encountered although there is no strong evidence utilizing carefully designed prospective studies with instrumental evaluation to determine aspiration and reflecting these practices to support clinical guidelines. We recommend caution if orally feeding on CPAP or HFNC due to the potential for oropharyngeal aspiration and that each child should have a clinical feeding evaluation by a trained dysphagia therapist with the opportunity for VFSS or FEES to support clinical care, until further strong evidence is available.

\section{Conclusion}

This systematic review examined oral feeding efficacy and safety for infants and children receiving nCPAP and HFNC. Variations in NIV definitions, small cohort numbers, a wide variety of study outcomes and poorly defined AEs impacted on the ability to conduct a metaanalysis. Findings are insufficient to conclude whether commencing oral feeding whilst on nCPAP or HFNC facilitates transition to full oral feeding without adverse events. Further research is warranted, including prospective studies with instrumental assessment of swallow safety, in particular on HFNC for which instrumental assessment has not yet been utilitied. This will assist in the future development of clinical guidelines and recommendations for best practice with these populations.

\footnotetext{
Abbreviations

nCPAP: Nasal continuous positive airway pressure; HFNC: High flow nasal cannula; LFNC: Low flow nasal cannula; NIV: Non-invasive ventilation; NICU: Neonatal intensive care unit; PICU: Pediatric intensive care unit; CNLD: Chronic neonatal lung disease; BPD: Bronchopulmonary dysplasia; AE: Adverse event; PMA: Post-menstrual age; GA: Gestational age; OPA: Oropharyngeal aspiration; VFSS: Videofluoroscopic swallow study; FEES: Fiberoptic endoscopic evaluation of swallowing; RCT: Randomised control trial; NPO: Nil per oral
}

\section{Acknowledgements}

We would like to thank Professor Sharon Mickan for her support in providing an Allied Health Research Clinical Backfill Grant (AC) and Melissa Lawrie, Director or Speech Pathology GCUH for her ongoing support and commitment to research.

\section{Authors' contributions}

KW and AC conceptualized and designed the study, completed data extraction and quality assessment. AC and SC performed abstract and full text screening. AC drafted the initial manuscript. KW, SC, ST and MC reviewed and revised the manuscript. All authors approved the final manuscript as submitted.

\section{Funding}

Gold Coast Hospital and Health Service Allied Health Research Clinical Backfill Scheme.

\section{Availability of data and materials}

All data generated or analysed during this study are included in this published article (and its supplementary information files).

\section{Ethics approval and consent to participate}

Not applicable.

\section{Consent for publication \\ Not applicable.}

\section{Competing interests}

The authors declare that they have no competing interests.

\section{Author details}

${ }^{1}$ Speech Pathology, Gold Coast University Hospital, Gold Coast Health, Gold Coast, Australia. ${ }^{2}$ Queensland Children's Hospital, Children's Health

Queensland, Brisbane, Australia. ${ }^{3}$ Library Services, Gold Coast University Hospital, Gold Coast Health, Gold Cost, Australia. ${ }^{4}$ Newborn Care Unit, Gold Coast University Hospital, Gold Coast Health, Gold Coast, Australia. ${ }^{5}$ Allied Health Sciences \& Menzies Health Institute Queensland Griffith University, Gold Coast, Australia. ${ }^{6}$ Allied Health Research Gold Coast Health, Gold Coast, Australia.

Received: 20 November 2020 Accepted: 5 January 2021

Published online: 17 February 2021

\section{References}

1. Wilkinson D, Andersen C, O'Donnell CP, De Paoli AG, Manley BJ. High flow nasal cannula for respiratory support in preterm infants. Cochrane Database Syst Rev. 2016;2:CD006405.

2. Lee $J H$, Rehder KJ, Williford L, Cheifetz IM, Turner DA. Use of high flow nasal cannula in critically ill infants, children, and adults: a critical review of the literature. Intensive Care Med. 2013;39(2):247-57.

3. Morley SL. Non-invasive ventilation in paediatric critical care. Paediatr Respir Rev. 2016;20:24-31.

4. Roberts $\mathrm{CL}$, Badgery-Parker T, Algert CS, Bowen JR, Nassar N. Trends in use of neonatal CPAP: a population-based study. BMC Pediatr. 2011;11:89.

5. Coletti KD, Bagdure DN, Walker LK, Remy KE, Custer JW. High-flow nasal cannula utilization in pediatric critical care. Respir Care. 2017;62(8):1023-9.

6. Milesi C, Essouri S, Pouyau R, Liet JM, Afanetti M, Portefaix A, et al. High flow nasal cannula (HFNC) versus nasal continuous positive airway pressure (nCPAP) for the initial respiratory management of acute viral bronchiolitis in young infants: a multicenter randomized controlled trial (TRAMONTANE study). Intensive Care Med. 2017;43(2):209-16.

7. Wilkinson DJ, Andersen CC, Smith K, Holberton J. Pharyngeal pressure with high-flow nasal cannulae in premature infants. J Perinatol. 2008;28(1):42-7.

8. Shetty S, Hunt K, Douthwaite A, Athanasiou M, Hickey A, Greenough A. High-flow nasal cannula oxygen and nasal continuous positive airway pressure and full oral feeding in infants with bronchopulmonary dysplasia. Arch Dis Child Fetal Neonatal Ed. 2016;101(5):F408-11.

9. Slain KN, Martinez-Schlurmann N, Shein SL, Stormorken A. Nutrition and high-flow nasal cannula respiratory support in children with bronchiolitis. Hosp Pediatr. 2017;7(5):256-62.

10. Dalgleish SR, Kostecky LL, Blachly N. Eating in "SINC": safe individualized nipple-feeding competence, a quality improvement project to explore infant-driven Oral feeding for very premature infants requiring noninvasive respiratory support. Neonatal Netw. 2016;35(4):217-27.

11. Dodrill P, Gosa M, Thoyre S, Shaker C, Pados B, Park J, et al. FIRST, DO NO HARM: a response to "Oral alimentation in neonatal and adult populations requiring high-flow oxygen via nasal cannula". Dysphagia. 2016;31(6):781-2.

12. Parke RL, Eccleston ML, McGuinness SP. The effects of flow on airway pressure during nasal high-flow oxygen therapy. Respir Care. 2011;56(8): 1151-5. 
13. Tracy MC, Cornfield DN. Children with bronchiolitis on high-flow nasal cannula: to feed or not feed, that is not the only question. Hosp Pediatr 2017;7(5):297-9

14. Ferrara L, Bidiwala A, Sher I, Pirzada M, Barlev D, Islam S, et al. Effect of nasal continuous positive airway pressure on the pharyngeal swallow in neonates. J Perinatol. 2017;37(4):398-403.

15. Nishino T, Sugimori K, Kohchi A, Hiraga K. Nasal constant positive airway pressure inhibits the swallowing reflex. Am Rev Respir Dis. 1989;140(5): 1290-3.

16. Leder SB, Siner JM, Bizzarro MJ, McGinley BM, Lefton-Greif MA. Oral alimentation in neonatal and adult populations requiring high-flow oxygen via nasal cannula. Dysphagia. 2016;31(2):154-9.

17. Bizzarro MJ, Lefton-Greif MA, McGinley BM, Siner JM. FIRST, "KNOW" HARM: response to letter to the editor. Dysphagia. 2016;31(6):783-5.

18. Canning A, Fairhurst $R$, Chauhan M, Weir KA. Oral feeding for infants and children receiving nasal continuous positive airway pressure and high-flow nasal cannula respiratory supports: a survey of practice. Dysphagia. 2020; 35(3):443-54.

19. Canning A, Clarke S, Thorning S, Chauhan M, Weir K. Does introducing oral feeding to infants and children requiring high flow oxygen or nCPAP facilitate full oral feeding without adverse effects?: PROSPERO: International prospective register of systematic reviews; 2016 [CRD42016039325:[Available from: https:// www.crd.york.ac.uk/prospero/display_record.php? RecordID=39325.

20. Covidence systematic review software. Veritas Health Innovation. Available from: Available at www.covidence.org. Accessed 29 Apr 2016.

21. Sterne JA, Hernan MA, Reeves BC, Savovic J, Berkman ND, Viswanathan M, et al. ROBINS-I: a tool for assessing risk of bias in non-randomised studies of interventions. BMJ. 2016;355:14919.

22. Sterne JAC, Savovic J, Page MJ, Elbers RG, Blencowe NS, Boutron I, et al. RoB 2: a revised tool for assessing risk of bias in randomised trials. BMJ. 2019:366:14898

23. Bapat R, Gulati IK, Jadcherla S. Impact of SIMPLE feeding quality improvement strategies on Aerodigestive milestones and feeding outcomes in BPD infants. Hosp Pediatr. 2019;9(11):859-66.

24. Dumpa V, Kamity R, Ferrara L, Akerman M, Hanna N. The effects of oral feeding while on nasal continuous positive airway pressure (NCPAP) in preterm infants. J Perinatol. 2020;40(6):909-15.

25. Glackin SJ, O'Sullivan A, George S, Semberova J, Miletin J. High flow nasal cannula versus NCPAP, duration to full oral feeds in preterm infants: a randomised controlled trial. Arch Dis Child Fetal Neonatal Ed. 2017;102(4): F329-F32.

26. Hanin M, Nuthakki S, Malkar MB, Jadcherla SR. Safety and efficacy of Oral feeding in infants with BPD on nasal CPAP. Dysphagia. 2015;30(2):121-7.

27. Jadcherla SR, Hasenstab KA, Sitaram S, Clouse BJ, Slaughter JL, Shaker R. Effect of nasal noninvasive respiratory support methods on pharyngeal provocation-induced aerodigestive reflexes in infants. Am J Physiol Gastrointest Liver Physiol. 2016;310(11):G1006-14.

28. Leroue MK, Good RJ, Skillman HE, Czaja AS. Enteral nutrition practices in critically ill children requiring noninvasive positive pressure ventilation. Pediatr Crit Care Med. 2017:18(12):1093-8.

29. Shadman KA, Kelly MM, Edmonson MB, Sklansky DJ, Nackers K, Allen A, et al. Feeding during high-flow nasal cannula for bronchiolitis: associations with time to discharge. J Hosp Med. 2019;14:E43-E8.

30. Shimizu D, Araki S, Kawamura M, Kuwamura M, Suga S, Miyake F, et al Impact of high flow nasal cannula therapy on Oral feeding in very low birth weight infants with chronic lung disease. J UOEH. 2019;41(2):131-8.

31. Sochet AA, McGee JA, October TW. Oral nutrition in children with bronchiolitis on high-flow nasal cannula is well tolerated. Hosp Pediatr. 2017;7(5):249-55.

32. Ross ES, Philbin MK. Supporting oral feeding in fragile infants: an evidencebased method for quality bottle-feedings of preterm, ill, and fragile infants. J Perinat Neonatal Nurs. 2011;25(4):349-57 quiz 58-9.

33. Leibel SL, Castro M, McBride T, Hassall K, Sarmiento K, Ye XY, et al. Comparison of continuous positive airway pressure versus high flow nasal cannula for Oral feeding preterm infants (CHOmP): randomized pilot study. J Matern Fetal Neonatal Med. 2020:1-7.

34. LaTuga MS, Mittelstaedt G, Moon JY, Kim M, Murray-Keane L, Si W, et al. Clinical characteristics of premature infants who orally feed on continuous positive airway pressure. Early Hum Dev. 2019;139:104833.

35. McGuinness, LA, Higgins, JPT. Risk-of-bias VISualization (robvis): An R package and Shiny web app for visualizing risk-of-bias assessments. Res Syn Meth. 2020; 1-7. https://doi.org/10.1002/jrsm.1411
36. Weir K, McMahon S, Barry L, Masters IB, Chang AB. Clinical signs and symptoms of oropharyngeal aspiration and dysphagia in children. Eur Respir J. 2009;33(3):604-11

37. DeMatteo C, Matovich D, Hjartarson A. Comparison of clinical and videofluoroscopic evaluation of children with feeding and swallowing difficulties. Dev Med Child Neurol. 2005:47(3):149-57.

38. Ross E, Browne J. Feeding outcomes in preterm infants after discharge from the neonatal intensive care unit: a systematic review. Newborn Infant Nurs Rev. 2013;13(2):87-93.

39. Amaizu N, Shulman R, Schanler R, Lau C. Maturation of oral feeding skills in preterm infants. Acta Paediatr. 2008:97(1):61-7.

40. Khoshoo V, Edell D. Previously healthy infants may have increased risk of aspiration during respiratory syncytial viral bronchiolitis. Pediatrics. 1999; 104(6):1389-90.

41. Pagliaro $\mathrm{CL}$, Buhler KE, Ibidi SM, Limongi SC. Dietary transition difficulties in preterm infants: critical literature review. J Pediatr. 2016;92(1):7-14.

42. Mizuno K, Nishida Y, Taki M, Hibino S, Murase M, Sakurai M, et al. Infants with bronchopulmonary dysplasia suckle with weak pressures to maintain breathing during feeding. Pediatrics. 2007;120(4):e1035-42.

43. Davis NL, Liu A, Rhein L. Feeding immaturity in preterm neonates: risk factors for oropharyngeal aspiration and timing of maturation. J Pediatr Gastroenterol Nutr. 2013:57(6):735-40.

44. Boesch RP, Daines C, Willging JP, Kaul A, Cohen AP, Wood RE, et al. Advances in the diagnosis and management of chronic pulmonary aspiration in children. Eur Respir J. 2006;28(4):847-61.

45. Wallis CRM. Assessing the role of Aspiration in Pediatric Lung Disease. Pediatr Allergy Immunol Pulmonol. 2012;25(3):132-42.

46. Mayfield S, Jauncey-Cooke J, Hough JL, Schibler A, Gibbons K, Bogossian F. High-flow nasal cannula therapy for respiratory support in children. Cochrane Database Syst Rev. 2014;3:CD009850.

47. Kubicka ZJ, Limauro J, Darnall RA. Heated, humidified high-flow nasal cannula therapy: yet another way to deliver continuous positive airway pressure? Pediatrics. 2008:121(1):82-8.

\section{Publisher's Note}

Springer Nature remains neutral with regard to jurisdictional claims in published maps and institutional affiliations.

\section{Ready to submit your research? Choose BMC and benefit from:}

- fast, convenient online submission

- thorough peer review by experienced researchers in your field

- rapid publication on acceptance

- support for research data, including large and complex data types

- gold Open Access which fosters wider collaboration and increased citations

- maximum visibility for your research: over $100 \mathrm{M}$ website views per year

At $\mathrm{BMC}$, research is always in progress.

Learn more biomedcentral.com/submissions 